\title{
Fuzzy Inverse Compactness
}

\author{
Halis Aygün, ${ }^{1}$ A. Arzu Bural, ${ }^{2}$ and S. R. T. Kudri ${ }^{3}$ \\ ${ }^{1}$ Department of Mathematics, Faculty of Arts and Sciences, Kocaeli University, Kocaeli 41380, Turkey \\ ${ }^{2}$ Department of Mathematics, Faculty of Education, Kocaeli University, Kocaeli 41380, Turkey \\ ${ }^{3}$ Department of Mathematics, Federal University of Parana, P.O. Box 019081, Curitiba, \\ PR 81531-990, Brazil
}

Correspondence should be addressed to Halis Aygün, halis@kou.edu.tr

Received 9 February 2007; Revised 25 November 2007; Accepted 14 January 2008

Recommended by Heinz Gumm

We introduce definitions of fuzzy inverse compactness, fuzzy inverse countable compactness, and fuzzy inverse Lindelöfness on arbitrary $L$-fuzzy sets in $L$-fuzzy topological spaces. We prove that the proposed definitions are good extensions of the corresponding concepts in ordinary topology and obtain different characterizations of fuzzy inverse compactness.

Copyright (C 2008 Halis Aygün et al. This is an open access article distributed under the Creative Commons Attribution License, which permits unrestricted use, distribution, and reproduction in any medium, provided the original work is properly cited.

\section{Introduction}

In ordinary topology, Matveev [1] has introduced a topological property called inverse compactness which is weaker than compactness and stronger than countable compactness. In $[1,2]$, inverse countable compactness and inverse Lindelöfness have been defined and studied.

A topological space $X$ is called inversely compact if and only if for every open cover $\beta$ of $X$, one can select a finite subcover $\gamma$ of $X$ which consists of the elements of $\beta$ or their complements (but of course $\gamma$ is prohibited to contain both $U$ and $X \backslash U$ for any $U \in \beta$ ).

In $L$-fuzzy topological spaces fuzzy compactness has been introduced by Warner and McLean [3] and extended to arbitrary L-fuzzy sets by Kudri [4].

In this paper, we initiate fuzzy inverse compactness in $L$-fuzzy topological spaces which is weaker than fuzzy compactness and introduce fuzzy inverse countable compactness and fuzzy inverse Lindelöfness. We prove that proposed definitions are good extensions of the corresponding notions in ordinary topology.

\section{Preliminaries}

We assume that the reader is familiar with the usual notations and most of the concepts of fuzzy topology and lattice theory. 
Throughout this paper $X$ and $Y$ will be nonempty ordinary sets and $L=L\left(\leq, \vee, \wedge,,^{\prime}\right)$ will denote a completely distributive lattice with a smallest element 0 and a largest element $1(0 \neq 1)$ and with an order-reversing involution $a \rightarrow a^{\prime}(a \in L) . \tau$ and $T$ will denote $L$-fuzzy topology and ordinary topology, respectively.

Definition 2.1 (see [5]). An element $p$ of a lattice $L$ is called prime if and only if $p \neq 1$ and whenever $a, b \in L$ with $a \wedge b \leq p$ then $a \leq p$ or $b \leq p$.

The set of all prime elements of a lattice $L$ will be denoted by $\operatorname{pr}(L)$.

Definition 2.2 (see [5]). An element $\alpha$ of a lattice $L$ is called coprime (or union irreducible) if and only if $\alpha \neq 0$ and whenever $a, b \in L$ with $\alpha \leq a \vee b$ then $\alpha \leq p$ or $\alpha \leq b$.

The set of all coprime elements of a lattice $L$ will be denoted by $M(L)$.

From the definitions we have that $p \in \operatorname{pr}(L) \Leftrightarrow p^{\prime} \in M(L)$.

Definition 2.3 (see [3]). Let $L$ be a complete lattice. A subset $U$ of $L$ is called Scott open if and only if it is an upper set and is inaccessible by directed joins, that is,

(a) if $a \in U$ and $a \leq b$, then $b \in U$;

(b) if $D$ is a directed subset of $L$ with $\bigvee D \in U$, then there is a $d \in D$ with $d \in U$.

The collection of all Scott-open subsets of $L$ is a topology on $L$ and is called Scott topology of $L$. The Scott topology of completely distributive lattice $L$ is generated by the sets of the form $\{x \in L \mid x \not \leq p\}$, where $p \in \operatorname{pr}(L)$. This means that the family $\{\{x \in L \mid x \not \leq p\} \mid p \in \operatorname{pr}(L)\}$ forms a base for Scott topology on $L$.

A function $f$ from $(X, T)$ to $L$ with its Scott topology is Scott continuous if and only if $f^{-1}(\{x \in X \mid x \not \leq p\}) \in T$ for every $p \in \operatorname{pr}(L)$.

Definition 2.4 (see [6]). An $L$-fuzzy topology on $X$ is a subset $\tau$ of $L^{X}$ satisfying the following properties:

(i) the constant functions 0 and 1 belong to $\tau$;

(ii) if $f, g \in \tau$, then $f \wedge g \in \tau$;

(iii) if $\left\{f_{i}: i \in J\right\} \subseteq \tau$, then $\bigvee_{i \in J} f_{i} \in \tau$.

The pair $(X, \tau)$ is called an $L$-fuzzy topological space. The elements of $\tau$ are called open $L$-fuzzy sets. An $L$-fuzzy set $h$ is said to be closed if and only if $h^{\prime} \in \tau$.

Definition 2.5 (see [6]). Let $(X, \tau)$ and $\left(Y, \tau^{*}\right)$ be $L$-fuzzy topological spaces. A function $\varphi$ : $(X, \tau) \rightarrow\left(Y, \tau^{*}\right)$ is called fuzzy continuous if and only if $\varphi^{-1}(g) \in \tau$ for every $g \in \tau^{*} .\left(\varphi^{-1}(g)=\right.$ $g(\varphi(x)))$.

Definition 2.6 (see [7]). Let $(X, T)$ be an ordinary topological space. The set of all Scott continuous functions from $(X, T)$ to $L$ with its Scott topology forms an $L$-fuzzy topology on $X$, which will be denoted by $\omega_{L}(T)$, that is,

$$
\omega_{L}(T)=\left\{f \in L^{X} \mid f:(X, T) \longrightarrow L \text { is Scott continuous }\right\} .
$$


Halis Aygün et al.

Definition 2.7 (see [7]). Let $(X, T)$ be an ordinary topological space. An L-fuzzy topological property $P_{f}$ is a "good extension" of a topological property $P$ if and only if the topological space $(X, T)$ has $P$ if and only if the induced $L$-fuzzy topological space $\left(X, \omega_{L}(T)\right)$ has $P_{f}$.

Definition 2.8 (see [4]). Let $(X, \tau)$ be an $L$-fuzzy topological space, $g \in L^{X}$ and $p \in L$. A collection $\beta=\left(f_{i}\right)_{i \in J}$ of open $L$-fuzzy sets is called a $p$-level open cover of the $L$-fuzzy set $g$ if and only if $\left(\bigvee_{i \in J} f_{i}\right)(x) \not \leq p$ for all $x \in X$ with $g(x) \geq p^{\prime}$.

If $g$ is the whole space $X(=1)$, then $\beta$ is called a $p$-level open cover of $X$.

Thus $\beta$ is called a $p$-level open cover of $X$ if and only if $\left(\bigvee_{f \in \beta} f\right)(x) \not \leq p$ for all $x \in X$.

Definition 2.9 (see [4]). Let $(X, \tau)$ be an $L$-fuzzy topological space and $g \in L^{X}$. The $L$-fuzzy set $g$ is said to be fuzzy compact if and only if every $p$-level open cover of $g$, where $p \in \operatorname{pr}(L)$, has a finite $p$-level subcover of $g$.

If $g$ is the whole space $X$, then $(X, \tau)$ is called fuzzy compact $L$-fuzzy topological space.

\section{Proposed definitions}

Definition 3.1. Let $X$ be nonempty set and let $\beta, \gamma \subseteq L^{X}$. $\beta$ is called a partial inversement of $\gamma$ if and only if $\beta$ and $\gamma$ can be indexed with the same index set, say $J: \beta=\left\{f_{i}: i \in J\right\}, \gamma=\left\{g_{i}: i \in J\right\}$ so that for every $i \in J$ either $f_{i}=g$ or $f_{i}=g_{i}^{\prime}$.

Definition 3.2. Let $(X, \tau)$ be an $L$-fuzzy topological space and $g \in L^{X} \cdot g$ is said to be fuzzy inversely compact if and only if every $p$-level open cover of $g$, where $p \in \operatorname{pr}(L)$, has a partial inversement which contains a finite $p$-level cover of $g$.

If $g$ is the whole space $X$, then $(X, \tau)$ is called fuzzy inverse compact $L$-fuzzy topological space.

This definition can be restated as follows. $g$ is fuzzy inverse compact if and only if for every $p$-level open cover $\beta=\left\{f_{i}: i \in J\right\}$ of $g$, where $p \in \operatorname{pr}(L)$, there exists a finite $p$-level subcover $\gamma=\left\{g_{i}: i \in J\right\}$ of $g$ such that there exist $g_{1}, g_{2}, \ldots, g_{n} \in \gamma:\left(\bigvee_{i=1}^{n} g_{i}\right)(x) \not \leq p$ for every $x \in X$ with $g(x) \geq p^{\prime}$, where for each $i=1,2, \ldots, n, g_{i}=f_{i}$, or $g_{i}=f_{i}^{\prime}$.

Clearly, every fuzzy compact $L$-fuzzy set is inversely fuzzy compact.

Definition 3.3. Let $(X, \tau)$ be an $L$-fuzzy topological space and $g \in L^{X} \cdot g$ is said to be fuzzy inversely countably compact if and only if for every countable $p$-level open cover of $g$, where $p \in \operatorname{pr}(L)$, has a partial inversement which contains a finite $p$-level cover of $g$.

If $g$ is the whole space, then we say that the $L$-fuzzy topological space $(X, \tau)$ is fuzzy inversely countably compact.

Definition 3.4. Let $(X, \tau)$ be an $L$-fuzzy topological space and $g \in L^{X} \cdot g$ is said to be fuzzy inversely Lindelöf if and only if every $p$-level open cover of $g$, where $p \in \operatorname{pr}(L)$, has a partial inversement which contains a countable $p$-level cover of $g$.

If $g$ is the whole space, then we say that the $L$-fuzzy topological space $(X, \tau)$ is fuzzy inversely Lindelöf. 
From the definitions, it can be easily verified that every fuzzy inversely compact $L$-fuzzy set $g$ is fuzzy inversely countably compact and fuzzy inversely Lindelöf.

\section{Other characterizations}

Definition 4.1. Let $\alpha \in L, \zeta \subseteq L^{X}$, and $g \in L^{X}$. $\zeta$ is called an $\alpha$-level centered family of $g$ if and only if for any $h_{1}, h_{2}, \ldots, h_{n} \in \zeta$ there exists $x \in X$ with $g(x) \geq \alpha$ such that $\left(h_{1} \wedge h_{2} \wedge \cdots \wedge h_{n}\right)(x) \geq \alpha$.

Theorem 4.2. Let $(X, \tau)$ be an $L$-fuzzy topological space and $g \in L^{X} . g$ is fuzzy compact if and only if for every $\alpha$-level centered family $\zeta$ of closed $L$-fuzzy sets, where $\alpha \in M(L)$, there exists $x \in X$ with $g(x) \geq \alpha$ such that $\left(\bigwedge_{h \in \zeta} h\right)(x) \geq \alpha$.

Proof

Necessity. Let $\alpha \in M(L)$ and let $\zeta$ be $\alpha$-level centered family of closed $L$-fuzzy sets such that for each $x \in X$ with $g(x) \geq \alpha$, there exists $h \in \zeta$ with $h(x) \nsupseteq \alpha$.

Then, $\left(\bigwedge_{h \in \zeta} h\right)(x) \not \leq \alpha$ for all $x \in X$ with $g(x) \geq \alpha$.

Hence $\left(\bigvee_{h \in \zeta} h^{\prime}\right)(x) \nsucceq p$ for all $x \in X$ with $g(x) \geq p^{\prime}$, where $p=\alpha^{\prime}$.

Thus, $\beta=\left\{h^{\prime}: h \in \zeta\right\}$ is a $p$-level open cover of $g$ that has no finite $p$-level subcover of $g$. In fact, if $h_{1}^{\prime}, h_{2}^{\prime}, \ldots, h_{n}^{\prime} \in \beta$, then, since $\zeta$ is $\alpha$-level centered, there exists $x \in X$ with $g(x) \geq \alpha$ and $\left(\bigwedge_{i=1}^{n} h_{i}\right)(x) \geq \alpha$; hence $\left(\bigvee_{i=1}^{n} h_{i}^{\prime}\right)(x) \leq p$.

Sufficiency. Suppose that $\beta$ is a $p$-level open cover of $g$ with no finite $p$-level subcover of $g$, where $p \in \operatorname{pr}(L)$. Then, $\zeta=\left\{f^{\prime}: f \in \beta\right\}$ is a collection of closed $L$-fuzzy sets. Moreover, $\zeta$ is an $\alpha$-level centered family of $g$, where $\alpha=p^{\prime}$. In fact, if $f_{1}^{\prime}, f_{2}^{\prime}, \ldots, f_{n}^{\prime} \in \zeta$, then there exists $x \in X$ with $g(x) \geq p^{\prime}=\alpha$ such that $\left(\bigvee_{i=1}^{n} f_{i}\right)(x) \leq p$; hence $\left(\bigwedge_{i=1}^{n} f_{i}^{\prime}\right)(x) \geq \alpha$.

By the hypothesis, there exists $x \in X$ with $g(x) \geq p^{\prime}$ with such that $\left(\bigwedge_{f \in \beta} f^{\prime}\right)(x) \geq p^{\prime}$; hence $\left(\bigvee_{f \in \beta} f\right)(x) \leq p$ which yields a contradiction.

Definition 4.3. Let $\zeta \subseteq L^{X}, g \in L^{X}$, and $\alpha \in L$.

$\zeta$ is called an $\alpha$-level independent family of $g$ if and only if for any finite $f_{1}, f_{2}, \ldots, f_{n}$, $g_{1}, g_{2}, \ldots, g_{m} \in \zeta$ there exists $x \in X$ with $g(x) \geq \alpha$ such that $\left(\bigwedge_{i=1}^{n} f_{i} \wedge \bigwedge_{i=1}^{m} g_{i}^{\prime}\right)(x) \geq \alpha$.

In other words, $\zeta$ is an $\alpha$-level independent family of $g$ if and only if for every nonempty finite partial inversement $\zeta^{*}$ of $\zeta$, there exists $x \in X$ with $g(x) \geq \alpha$ and $\left(\bigwedge_{h \in \zeta^{*}} h\right)(x) \geq \alpha$.

Theorem 4.4. Let $(X, \tau)$ be an $L$-fuzzy topological space and $g \in L^{X}$. $g$ is fuzzy inversely compact if and only if for every $\alpha$-level independent family $\zeta$ of closed L-fuzzy sets, where $\alpha \in M(L)$, there exists $x \in X$ with $g(x) \geq \alpha$ and $\left(\bigwedge_{h \in \zeta} h\right)(x) \geq \alpha$.

Proof

Necessity. Let $\alpha \in M(L)$. Suppose that $\zeta$ is an $\alpha$-level independent family of $L$-fuzzy sets, such that $\left(\bigwedge_{h \in \zeta} h\right)(x) \ngtr \alpha$ for every $x \in X$ with $g(x) \geq \alpha$.

Then, $\left(\bigvee_{h \in \zeta} h^{\prime}\right)(x) \not \leq \alpha^{\prime}$ for all $x \in X$ with $g(x) \geq \alpha$.

Hence, $\beta=\left\{h^{\prime}: h \in \zeta\right\}$ is a $p$-level open cover of $g$, where $p=\alpha^{\prime}$.

By the fuzzy inverse compactness of $g$, there is a partial inversement $\gamma$ of $\beta$ which contains a finite $p$-level subcover of $g$.

Let $\gamma=\left\{g_{i}: i \in J\right\}$ and $\beta=\left\{h_{i}^{\prime}: i \in J, h_{i} \in \zeta\right\}$.

Then, there exists $g_{1}, g_{2}, \ldots, g_{n} \in \gamma$ such that $\left(\bigvee_{i=1}^{n} g_{i}\right)(x) \not \leq p$ for all $x \in X$ with $g(x) \geq p^{\prime}$, where for each $i \in\{1,2, \ldots, n\}, g_{i}=h_{i}$, or $g_{i}=h_{i}^{\prime}$. 
Let $g_{1}=h_{1}^{\prime}, g_{2}=h_{2}^{\prime}, \ldots, g_{k}=h_{k}^{\prime}, g_{k+1}=h_{k+1}, g_{k+2}=h_{k+2}, \ldots, g_{n}=h_{n}$. Then $g_{1}^{\prime}, g_{2}^{\prime}$, $\ldots, g_{k^{\prime}}^{\prime} g_{k+1}, \ldots, g_{n} \in \zeta$. Since $\zeta$ is an $\alpha$-level independent family of $g$, there exists $x \in X$ with $g(x) \geq \alpha$ such that

$$
\left(\bigwedge_{i=1}^{k} g_{i}^{\prime} \wedge \bigwedge_{i=k+1}^{n} g_{i}^{\prime}\right)(x)=\left(\bigwedge_{i=1}^{n} g_{i}^{\prime}\right)(x) \geq \alpha
$$

Hence, there exists $x \in X$ with $g(x) \geq p^{\prime}$ such that $\left(\bigvee_{i=1}^{n} g_{i}\right)(x) \leq p$, which yields a contradiction.

Sufficiency. Suppose that $\beta$ is a $p$-level open cover of $g$ with no partial inversement which contains a finite $p$-level subcover of $g$, where $p \in \operatorname{pr}(L)$. Then, $\zeta=\left\{f^{\prime}: f \in \beta\right\}$ is a collection of closed $L$-fuzzy sets. Furthermore, $\zeta$ is an $\alpha$-level independent family of $g$, where $\alpha=p^{\prime}$. In fact, if $f_{1}^{\prime}, f_{2}^{\prime}, \ldots, f_{k}^{\prime}, f_{k+1}^{\prime}, \ldots, f_{n}^{\prime} \in \zeta$, then by the assumption, there exists $x \in X$ with $g(x) \geq p^{\prime}$ and $\left(\bigvee_{i=1}^{k} f_{i} \vee \bigvee_{i=k+1}^{n} f_{i}^{\prime}\right)(x) \leq p$; hence $\left(\bigwedge_{i=1}^{k} f_{i}^{\prime} \wedge \bigwedge_{i=k+1}^{n} f_{i}\right)(x) \geq \alpha$.

By the hypothesis, there exists $z \in X$ with $g(z) \geq \alpha$ and $\left(\bigwedge_{f \in \beta} f^{\prime}\right)(z) \geq \alpha$; hence $\left(\bigvee_{f \in \beta} f\right)(z) \leq p$, which yields a contradiction.

Theorem 4.5. Let $(X, \tau)$ be an L-fuzzy topological space and $g \in L^{X} . g$ is fuzzy inversely countably compact if and only if for every countable $\alpha$-level independent family $\zeta$ of closed L-fuzzy sets, where $\alpha \in M(L)$, there exists $x \in X$ with $g(x) \geq \alpha$ and $\left(\bigwedge_{h \in \beta} h\right)(x) \geq \alpha$.

Proof. This is similar to Theorem 4.4.

Definition 4.6. Let $\xi \subseteq L^{X}$ and $p \in \operatorname{pr}(L)$. $\xi$ is said to have the finite union property (for short FUP) in $G=\left\{x \in X: g(x) \geq p^{\prime}\right\}$ if and only if for any finite $f_{1}, f_{2}, \ldots, f_{k}, g_{1}, g_{2}, \ldots, g_{n} \in \xi$, there exists $x \in G$ with $\left(\bigvee_{i=1}^{k} f_{i} \vee \bigvee_{i=1}^{n} g_{i}^{\prime}\right)(x) \leq p$.

Theorem 4.7. Let $(X, \tau)$ be an $L$-fuzzy topological space and $g \in L^{X}$. $g$ is fuzzy inversely compact if and only if no $\xi \subseteq \tau$ with the FUP in $G$ satisfies $\left(\bigvee_{f \in \xi} f\right)(x) \not \leq p$ for all $x \in G$.

Proof

Necessity. Suppose that $\xi \subseteq \tau$ has the FUP in G such that $\left(\bigvee_{f \in \xi} f\right)(x) \not \leq p$ for all $x \in G$.

Then, $\zeta=\left\{f^{\prime}: f \in \xi\right\}$ is an $\alpha$-level independent family of closed $L$-fuzzy sets, where $\alpha=p^{\prime}$. From Theorem 4.4, there exists $x \in X$ with $g(x) \geq \alpha$ and $\left(\bigwedge_{f \in \zeta} f^{\prime}\right)(x) \geq \alpha$; hence $\left(\bigvee_{f \in \xi} f\right)(x) \leq p$ which yields a contradiction.

Sufficiency. Suppose that $g$ is not fuzzy inverse compact. Then, there is an $\alpha$-level independent family $\zeta$ of closed $L$-fuzzy sets such that $\left(\bigwedge_{h \in \zeta} h\right)(x) \nsupseteq \alpha$ for all $x \in X$ with $g(x) \geq \alpha$, where $\alpha \in M(L)$. Hence $\left(\bigvee_{h \in \zeta} h^{\prime}\right)(x) \not \leq p$ for all $x \in X$ with $g(x) \geq p^{\prime}$, where $p=\alpha^{\prime}$. Moreover, $\xi=\left\{h^{\prime}: h \in \zeta\right\}$ is a family of open $L$-fuzzy sets having the FUP in $G$. This completes the proof.

\section{Some properties}

The next theorem shows that fuzzy inverse compactness is a good extension of inverse compactness in general topology.

Theorem 5.1. Let $(X, T)$ be an ordinary topological space. $(X, T)$ is inversely compact if and only if the $L$-fuzzy topological space $\left(X, \omega_{L}(T)\right)$ is fuzzy inversely compact. 
Proof

Sufficiency. Let $\alpha \in M(L)$ and $\zeta$ be an $\alpha$-level independent family of closed subsets of $X$. Then, $\zeta^{*}=\left\{\chi_{A}: A \in \zeta\right\}$ is a family of closed $L$-fuzzy sets. Furthermore, $\zeta^{*}$ is an $\alpha$-level independent family of $X$. In fact, if $\chi_{A_{1}}, \chi_{A_{2}}, \ldots, \chi_{A_{n}}, \chi_{B_{1}, \ldots, X_{B_{m}} \in \zeta^{*}}$, then since $\zeta$ is independent, $\bigcap_{i=1}^{n} A_{i} \cap$ $\bigcap_{i=1}^{m} B_{i}^{\prime} \neq \phi$. Hence $\left(\bigwedge_{i=1}^{n} X_{A_{i}} \wedge \bigwedge_{i=1}^{m} X_{B_{i}}^{\prime}\right)=X \bigcap_{i=1}^{n} A_{i} \cap \bigcap_{i=1}^{m} B_{i}^{\prime} \neq 0$. So, there is $x \in X$ such that $\left(\bigwedge_{i=1}^{n} X_{A_{i}} \wedge\right.$ $\left.\bigwedge_{i=1}^{m} X_{B_{i}}^{\prime}\right)(x)=1 \geq \alpha$. Since $\left(X, \omega_{L}(T)\right)$ is fuzzy inversely compact, there exists $z \in X$ with $\left(\bigwedge_{A \in \zeta} X_{A}\right)(z) \geq \alpha$; hence $\left(\bigwedge_{A \in \zeta} X_{A}\right)(z)=\left(\chi_{\cap_{A \in \zeta} A}\right)(z)=1$ and therefore $z \in \bigcap_{A \in \zeta} A$. Hence $(X, T)$ is inversely compact.

Necessity. Let $p \in \operatorname{pr}(L)$ and $\beta=\left(f_{i}\right)_{i \in J}$ be a $p$-level open cover of $X$ consisting of basic open $L$-fuzzy sets in $\left(X, \omega_{L}(T)\right)$. Then

$$
f_{i}(x)= \begin{cases}e_{i} \in L ; & x \in A_{i} \in T, \\ 0 ; & x \notin A_{i}\end{cases}
$$

(for each $i \in J)$ and $\left(\bigvee_{i \in J} f_{i}\right)(x) \not \leq p$ for all $x \in X$.

Let $\zeta=\left\{A_{i} \subseteq X: \exists i \in J\right.$ with $e_{i} \not \leq p$ and $\left.f_{i} \in \beta\right\}$.

It is clear that $\zeta$ is an open cover of $X$ in $(X, T)$. Due to inverse compactness of $(X, T)$, there exists a partial inversement $\zeta^{*}$ of $\zeta$ which contains a finite cover of $X$.

Let $\zeta^{*}=\left\{B_{i}: i \in J\right\}$. Then there exist $B_{1}, B_{2}, \ldots, B_{n} \in \zeta^{*}$ such that $X=\bigcup_{i=1}^{n} B_{i}$, where $B_{i}=A_{i}$ and $B_{i}=A_{i}^{\prime}$ (for each $i \in\{1,2, \ldots, n\}$ ).

(i) If $B_{i}=A_{i}$ for all $i \in\{1,2, \ldots, n\}$, then $\beta^{*}=\left\{f_{1}, f_{2}, \ldots, f_{n}\right\}$ is a finite partial inversement of $\beta$. $\beta^{*}$ is also a $p$-level subcover of $X$ in $\left(X, \omega_{L}(T)\right)$. Hence, $\left(X, \omega_{L}(T)\right)$ is fuzzy inversely compact.

(ii) Suppose that there exists $i_{0} \in\{1,2, \ldots, n\}$ such that $B_{i_{0}}=A_{i_{0}}^{\prime}$.

Let $\beta^{*}=\left\{f_{1}, f_{2}, \ldots, f_{i_{0}-1}, f_{i_{0}}^{\prime}, \ldots, f_{n}^{\prime}\right\}$. Obviously, $\beta^{*}$ is a partial inversement of $\beta$. Furthermore, $\beta^{*}$ is a $p$-level subcover of $X$ in $\left(X, \omega_{L}(T)\right)$. In fact, let $x \in X$. Since $X=\bigcup_{i=1}^{n} B_{i}=$ $B_{i_{0}} \cup \bigcup_{i=1, i \neq i_{0}}^{n} B_{i} \Rightarrow x \in B_{i_{0}}$ or $x \in \bigcup_{i=1, i \neq i_{0}}^{n} B_{i} \Rightarrow x \in A_{i_{0}}^{\prime}$ or $x \in \bigcup_{i=1, i \neq i_{0}}^{n} A_{i}$.

$\left(1^{\circ}\right)$ If $x \in A_{i_{0}}^{\prime}$, then $f_{i_{0}}^{\prime}(x)=1 \not \leq p$. Hence $\left(f_{i_{0}}^{\prime}(x) \vee \bigvee_{i=1, i \neq i_{0}}^{n} f_{i}\right)(x) \not \leq p$.

$\left(2^{\circ}\right)$ If $x \notin A_{i_{0}}^{\prime}$, then there is $i \in\{1,2, \ldots, n\}-\left\{i_{0}\right\}$ such that $x \in A_{i}$. Hence $f_{i}(x)=$ $e_{i} \not \leq p$ and therefore $\left(f_{i_{0}}^{\prime} \vee \bigvee_{i=1, i \neq i_{0}}^{n} f_{i}\right)(x) \not \leq p$. Consequently, $\beta^{*}$ is $p$-level subcover of $X$ in $\left(X, \omega_{L}(T)\right)$.

Theorem 5.2 (the goodness of fuzzy inverse countable compactness). Let $(X, T)$ be an ordinary topological space. $(X, T)$ is inversely countably compact if and only if the L-fuzzy topological space $\left(X, \omega_{L}(T)\right)$ is fuzzy inversely countably compact.

Proof. This is similar to the proof of the goodness of fuzzy inverse compactness.

Theorem 5.3 (the goodness of fuzzy inverse Lindelöfness). Let $(X, T)$ be an ordinary topological space. $(X, T)$ is an inverse Lindelöf space if and only if the L-fuzzy topological space $\left(X, \omega_{L}(T)\right)$ is fuzzy inverse Lindelöf space.

Proof. This is similar to the proof of the goodness of fuzzy inverse compactness.

Theorem 5.4. Let $(X, \tau)$ be an L-fuzzy topological space. If $g \in L^{X}$ is fuzzy inversely compact and $h \in L^{X}$ closed, then $g \wedge h$ is also fuzzy inversely compact. 
Halis Aygün et al.

Proof. Let $p \in \operatorname{pr}(L)$ and let $\beta=\left(f_{i}\right)_{i \in J} \subseteq L^{X}$ be a $p$-level open cover of $g \wedge h$; that is, $\left(\bigvee_{i \in J} f_{i}\right)(x) \not \leq$ $p$ for all $x \in X$ with $(g \wedge h)(x) \geq p^{\prime}$. So $\beta^{*}=\left(f_{i}\right)_{i \in J} \cup\left\{h^{\prime}\right\}$ is $p$-level family of $g$. In fact, if

$$
\begin{aligned}
h(x) \geq p^{\prime} & \Longrightarrow\left(\bigvee_{i \in J} f_{i}\right)(x) \not \leq p \quad \forall x \in X \text { with }(g \wedge h)(x) \geq p^{\prime} \\
& \Longrightarrow\left(\bigvee_{k \in \beta^{*}} k\right)(x)=\left(\bigvee_{i \in J} f_{i}\right)(x) \vee h^{\prime}(x) \not \leq p \\
h(x) \ngtr p^{\prime} & \Longrightarrow h^{\prime}(x) \not \leq p \Longrightarrow\left(\bigvee_{k \in \beta^{*}} k\right)(x) \not \leq p .
\end{aligned}
$$

Since $g$ is fuzzy inversely compact, $\beta^{*}$ has a partial inversement $\gamma^{*}$ which contains a finite $p$-level subcover of $g$.

Let $\gamma^{*}=\left\{g_{i}: i \in J\right\} \cup\left\{h^{\prime}\right\}$ and $\beta^{*}=\left\{f_{i}: i \in J\right\} \cup\left\{h^{\prime}\right\}$.

Then there exists $g_{1}, g_{2}, \ldots, g_{n} \in \gamma^{*}$ such that $\left(\bigvee_{i=1}^{n} g_{i} \vee h^{\prime}\right)(x) \not \leq p$ for all $x \in X$ with $g(x) \geq p^{\prime}$ where for each $i \in\{1,2, \ldots, n\}, g_{i}=f_{i}$ or $g_{i}=f_{i}^{\prime}$.

So $\left(\bigvee_{i=1}^{n} g_{i}\right)(x) \not \leq p$ for all $x \in X$ with $(g \wedge h)(x) \geq p^{\prime}$. In fact $x \in X$ with $(g \wedge h)(x) \geq p \Rightarrow$ $g(x) \geq p^{\prime}$ and $h(x) \geq p^{\prime}$,

$$
\begin{aligned}
g(x) \geq p^{\prime} & \Longrightarrow\left(\bigvee_{i=1}^{n} g_{i} \vee h^{\prime}\right)(x) \not \leq p \\
& \Longrightarrow\left(\bigvee_{i=1}^{n} g_{i}\right)(x) \vee h^{\prime}(x) \not \leq p \\
& \Longrightarrow\left(\bigvee_{i=1}^{n} g_{i}\right)(x) \not \leq p .
\end{aligned}
$$

Corollary 5.5. Let $(X, \tau)$ be an L-fuzzy topological space. If $g$ is a fuzzy inversely compact L-fuzzy set and $h$ is a closed L-fuzzy set with $h \leq g$, then $h$ is fuzzy inversely compact as well.

Proof. This follows directly from the previous theorem.

Corollary 5.6. Let $(X, \tau)$ be an L-fuzzy topological space. If $(X, \tau)$ is fuzzy inversely compact, then every closed L-fuzzy set on $X$ is fuzzy inversely compact.

Proof. This follows from Theorem 5.4.

Proposition 5.7. Let $(X, \tau)$ be an L-fuzzy topological space and $g, h \in L^{X}$ with $h \leq g$. If $g$ is fuzzy inversely countably compact (fuzzy inversely Lindelöf) and $h$ is closed, then $h$ is fuzzy inversely countably compact (fuzzy inversely Lindelöf).

Proof. This is similar to Theorem 5.4.

Theorem 5.8. Let $(X, \tau)$ be an $L$-fuzzy topological space and $g, h \in L^{X}$. If $g$ and $h$ are fuzzy inversely compact, then $g \vee h$ is fuzzy inversely compact as well. 
Proof. Suppose that $g \vee h$ is not fuzzy inverse compact.

Then, there exists an $\alpha$-level independent family $\zeta$ of $g \vee h$ consisting of closed $L$-fuzzy sets such that $\left(\bigwedge_{f \in \zeta} f\right)(x) \geq \alpha$ for all $x \in X$ with $(g \vee h)(x) \geq \alpha$, where $\alpha \in M(L)$.

Since $\alpha \in M(L)$ we have $(g \vee h)(x) \geq \alpha \Leftrightarrow g(x) \geq \alpha$ or $h(x) \geq \alpha$.

Let $f_{1}, f_{2}, \ldots, f_{k}, f_{k+1}, \ldots, f_{n} \in \zeta$. Since $\zeta$ is an $\alpha$-level independent family of $g \vee h$, there exists $x \in X$ with $(g \vee h)(x) \geq \alpha$ and $\left(\bigwedge_{i=1}^{k} f_{i} \wedge \bigwedge_{i=k+1}^{n} f_{i}^{\prime}\right)(x) \geq \alpha$. That is, there exists $x \in X$ with $g(x) \geq \alpha$ or $h(x) \geq \alpha$ such that $\left(\bigwedge_{i=1}^{k} f_{i} \wedge \bigwedge_{i=k+1}^{n} f_{i}^{\prime}\right)(x) \geq \alpha$.

Assume that $g(x) \geq \alpha$. Then, $\zeta$ is $\alpha$-level independent family of $g$ consisting of closed L-fuzzy sets. Furthermore, $\left(\bigwedge_{f \in \zeta} f\right)(x) \nsupseteq \alpha$ for all $x \in X$ with $g(x) \geq \alpha$.

So, $g$ is not fuzzy inversely compact.

Proposition 5.9. Let $(X, \tau)$ be an $L$-fuzzy topological space and $g, h \in L^{X}$. If $g$ and $h$ are fuzzy inversely countably compact (fuzzy inversely Lindelöf), then $g \vee h$ is fuzzy inversely countably compact (fuzzy inversely Lindelöf).

Proof. This is similar to Theorem 5.8.

Theorem 5.10. Let $(X, \tau)$ and $\left(Y, \tau^{*}\right)$ be L-fuzzy topological spaces and let $\varphi:(X, \tau) \rightarrow\left(Y, \tau^{*}\right)$ be a fuzzy continuous function such that $\varphi^{-1}(y)$ is finite for every $y \in Y$. If $g \in L^{X}$ is fuzzy inversely compact in $(X, \tau)$, then $\varphi(g) \in L^{Y}$ is fuzzy inversely compact in $\left(Y, \tau^{*}\right)$, where $\varphi(g)(y)=\bigvee_{x \in \varphi^{-1}(y)} g(x)$.

Proof. Let $\alpha \in M(L)$ and $\zeta$ is an $\alpha$-level independent family of $\varphi(g)$ consisting of closed $L$-fuzzy sets in $\left(Y, \tau^{*}\right)$. Let $\zeta^{*}=\left\{\varphi^{-1}(h): h \in \zeta\right\}$.

Since $\varphi$ is fuzzy continuous, $\zeta^{*}$ is a family of closed $L$-fuzzy sets in $(X, \tau)$. Furthermore, $\zeta^{*}$ is an $\alpha$-level independent family of $g$. In fact, if $\varphi^{-1}\left(h_{1}\right), \varphi^{-1}\left(h_{2}\right), \ldots, \varphi^{-1}\left(h_{k}\right)$, $\varphi^{-1}\left(h_{k+1}\right), \ldots, \varphi^{-1}\left(h_{n}\right) \in \zeta^{*}$, then there exists $y \in Y$ with $\varphi(g)(y) \geq \alpha$ and $\left(\bigwedge_{i=1}^{k} h_{i} \wedge \bigwedge_{i=k+1}^{n} h_{i}^{\prime}\right)(y) \geq$ $\alpha$ because $\zeta$ is an $\alpha$-level independent family of $\varphi(g) \cdot \varphi(g)(y)=\bigvee_{x \in \varphi^{-1}(y)} g(x) \geq \alpha$ implies that there exists $x \in X$ with $g(x) \geq \alpha$ and $\varphi(x)=y$ because $\alpha \in M(L)$ and $\varphi^{-1}(y)$ is finite. Hence, $\left(\bigwedge_{i=1}^{k} \varphi^{-1}\left(h_{i}\right) \wedge \bigwedge_{i=k+1}^{n} \varphi^{-1}\left(h_{i}^{\prime}\right)\right)(x)=\left(\bigwedge_{i=1}^{k} h_{i} \wedge \bigwedge_{i=k+1}^{n} h_{i}^{\prime}\right)(\varphi(x)) \geq \alpha$. From the fuzzy inverse compactness of $g$, there exists $z \in X$ with $g(z) \geq \alpha$ and $\left(\bigwedge_{h \in \zeta} \varphi^{-1}(h)\right)(z) \geq \alpha$. Then, $\left(\bigwedge_{h \in \zeta} h\right)(\varphi(z)) \geq \alpha$. This means that $\varphi(g)$ is fuzzy inversely compact in $\left(Y, \tau^{*}\right)$.

Theorem 5.11. Let $(X, \tau)$ and $\left(Y, \tau^{*}\right)$ be L-fuzzy topological spaces and let $\varphi:(X, \tau) \rightarrow\left(Y, \tau^{*}\right)$ be a fuzzy continuous function such that $\varphi^{-1}(y)$ is finite for every $y \in Y$. If $g \in L^{X}$ is fuzzy inversely countable compact (fuzzy inversely Lindelöf) in $(X, \tau)$, then $\varphi(g) \in L^{Y}$ is fuzzy inversely countably compact (fuzzy inversely Lindelöf) in $\left(Y, \tau^{*}\right)$.

Proof. This is similar to the proof of Theorem 5.10 .

\section{References}

[1] M. V. Matveev, "Inverse compactness," Topology and Its Applications, vol. 62, no. 2, pp. 181-191, 1995.

[2] V. I. Malykhin and M. V. Matveev, Inverse Compactness Versus Compactness, vol. 767 of Annals New York Academy of Science, New York Academy of Science, New York, NY, USA, 1995.

[3] M. W. Warner and R. G. McLean, "On compact Hausdorff L-fuzzy spaces," Fuzzy Sets and Systems, vol. 56, no. 1, pp. 103-110, 1993.

[4] S. R. T. Kudri, “Compactness in L-fuzzy topological spaces,” Fuzzy Sets and Systems, vol. 67, no. 3, pp. 329-336, 1994. 
Halis Aygün et al.

[5] G. Gierz, K. H. Hofmann, K. Keimel, J. D. Lawson, M. W. Mislove, and D. S. Scott, A Compendium of Continuous Lattices, Springer, Berlin, Germany, 1980.

[6] C. L. Chang, "Fuzzy topological spaces," Journal of Mathematical Analysis and Applications, vol. 24, no. 1, pp. 182-190, 1968.

[7] M. W. Warner, "Fuzzy topology with respect to continuous lattices," Fuzzy Sets and Systems, vol. 35, no. 1, pp. 85-91, 1990. 


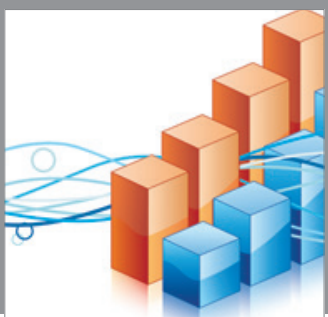

Advances in

Operations Research

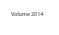

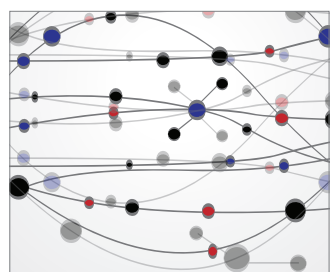

\section{The Scientific} World Journal
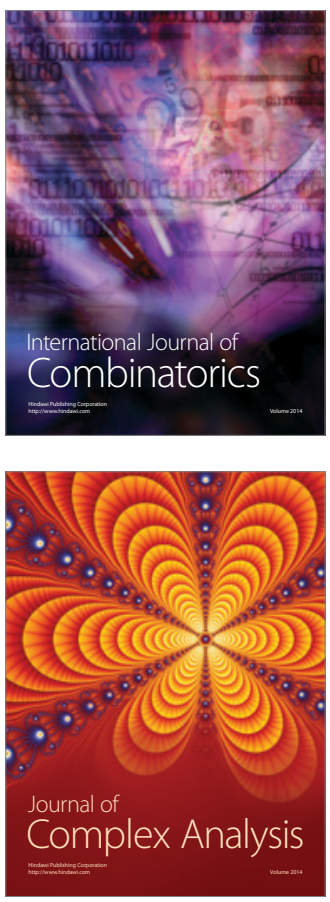

International Journal of

Mathematics and

Mathematical

Sciences
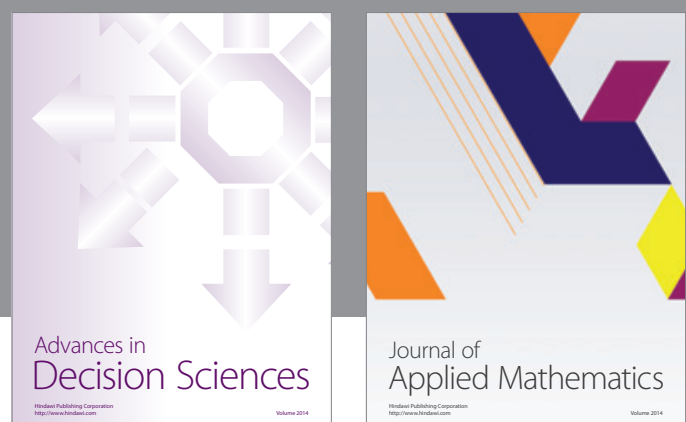

Journal of

Applied Mathematics
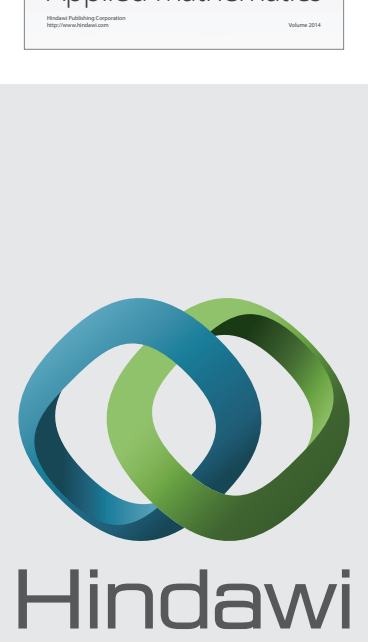

Submit your manuscripts at http://www.hindawi.com
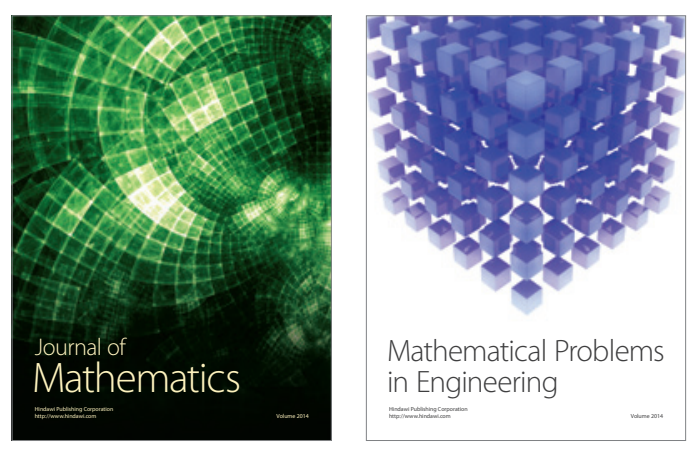

Mathematical Problems in Engineering
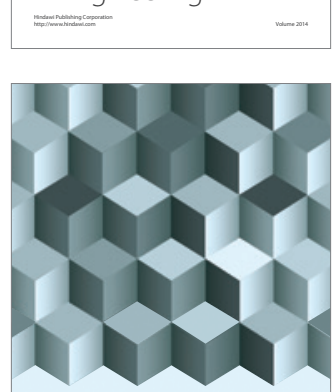

Journal of

Function Spaces
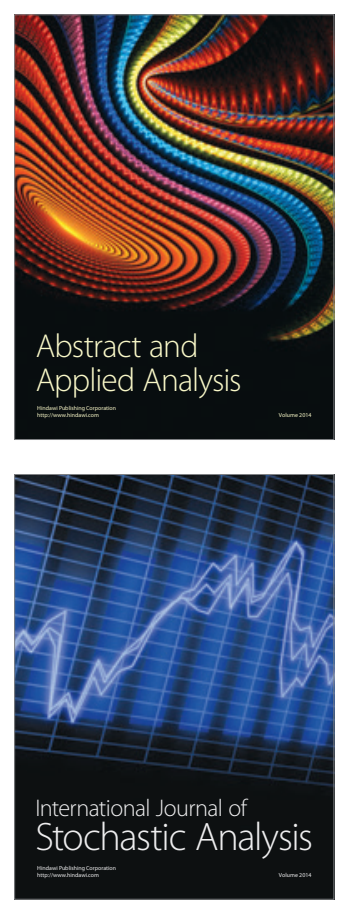

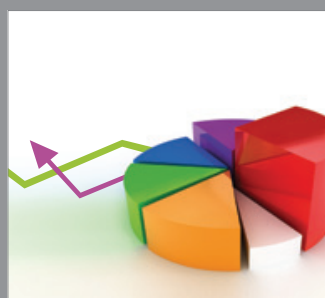

ournal of

Probability and Statistics

Promensencen
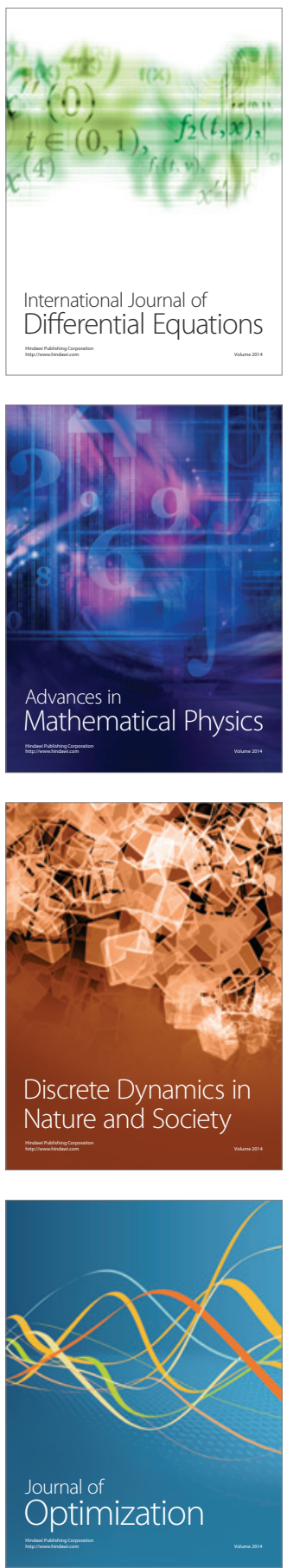